OPEN ACCESS

Edited by:

Vijayakumar Velu,

Emory University, United States

Reviewed by:

Saikat Boliar,

Cornell University, United States

Nicolas HUOT,

Institut Pasteur, France

Maud Mavigner,

Emory University, United States

*Correspondence:

Siddappa N. Byrareddy

sid.byrareddy@unmc.edu

Specialty section:

This article was submitted to

Viral Immunology,

a section of the journal

Frontiers in Immunology

Received: 01 July 2021 Accepted: 22 September 2021 Published: 13 October 2021

Citation:

Olwenyi OA, Johnson SD, Pandey K

Thurman M, Acharya A, Buch SJ,

Fox HS, Podany AT, Fletcher CV and

Byrareddy SN (2021) Diminished

Peripheral CD29 hi Cytotoxic CD4+ T

Cells Are Associated With Deleterious

Effects During SIV Infection.

Front. Immunol. 12:734871.

doi: 10.3389/fimmu.2021.734871

\section{Diminished Peripheral CD29hi Cytotoxic CD4+ T Cells Are Associated With Deleterious Effects During SIV Infection}

\author{
Omalla A. Olwenyi ${ }^{1,2}$, Samuel D. Johnson ${ }^{1,2}$, Kabita Pandey ${ }^{1,2}$, Michellie Thurman ${ }^{1}$, \\ Arpan Acharya ${ }^{1}$, Shilpa J. Buch ${ }^{1}$, Howard S. Fox ${ }^{3}$, Anthony T. Podany ${ }^{4}$, \\ Courtney V. Fletcher ${ }^{4}$ and Siddappa N. Byrareddy ${ }^{1,5,6,7 *}$
}

\footnotetext{
${ }^{1}$ Department of Pharmacology and Experimental Neuroscience, University of Nebraska Medical Center, Omaha, NE, United States, ${ }^{2}$ Department of Pathology and Microbiology, University of Nebraska Medical Center, Omaha, NE, United States, ${ }^{3}$ Department of Neurological Sciences, University of Nebraska Medical Center, Omaha, NE, United States, ${ }^{4}$ Antiviral Pharmacology Laboratory, Center for Drug Discovery, University of Nebraska Medical Center (UNMC), Omaha, NE, United States, ${ }^{5}$ Department of Genetics, Cell Biology, and Anatomy, University of Nebraska Medical Center, Omaha, NE, United States, ${ }^{6}$ Department of Biochemistry and Molecular Biology, University of Nebraska Medical Center, Omaha, NE, United States, ${ }^{7}$ Division of Clinical Microbiology, Department of Laboratory Medicine, Karolinska Institute, Stockholm, Sweden
}

Cytotoxic CD4+ T cells (CD4+ CTLs) limit HIV pathogenesis, as evidenced in elite controllers (a subset of individuals who suppress the virus without the need for therapy). CD4+ CTLs have also been shown to kill HIV-infected macrophages. However, little is known about their contribution towards HIV persistence, how they are affected following exposure to immune modulators like morphine, and what factors maintain their frequencies and function. Further, the lack of robust markers to identify CD4+ CTLs in various animal models limits understanding of their role in HIV pathogenesis. We utilized various PBMC samples obtained from SIV infected and CART treated rhesus macaques exposed to morphine or saline and subjected to flow cytometry evaluations. Thereafter, we compared and correlated the expression of CD4+CTL-specific markers to viral load and viral reservoir estimations in total CD4+ T cells. We found that CD29 could be reliably used as a marker to identify CD4+ CTLs in rhesus macaques since CD29hi CD4+ T cells secrete higher cytotoxic and proinflammatory cytokines following PMA/ionomycin or gag stimulation. In addition, this immune cell subset was depleted during untreated SIV infection. Strikingly, we also observed that early initiation of cART reconstitutes depleted CD29hi CD4+ T cells and restores their function. Furthermore, we noted that morphine exposure reduced the secretion of proinflammatory cytokines/cytotoxic molecules in CD29hi CD4+ T cells. Lastly, increased functionality of CD29hi CD4+ T cells as depicted by elevated levels of either IL-21 or granzyme B hi T Bet+ gag specific responses were linked to limiting the size of the replication-competent reservoir during cART treatment. Collectively, our data suggest that CD4+ CTLs are crucial in limiting SIV pathogenesis and persistence.

Keywords: CD4+ CTLs, SIV, morphine, reservoirs, CD29+ CD4+ T cells, biomarker 


\section{INTRODUCTION}

The proposed excision of latently infected HIV cells in a second person with HIV infection offers renewed optimism towards a cure that would usher an end to the AIDS crisis $(1,2)$. Although great strides have been undertaken, the cellular responses associated with sustaining persistence versus viral eradication remain poorly understood (3-5). HIV-infected individuals worldwide live different lifestyles stemming from varied cultural and sexual practices, co-exposures to other pathogens, and comorbid substance abuse not limited to the illicit use of drugs like morphine, cocaine, and heroin (6-11). Collectively, these behaviors differentially impact the body's immune response, alter disease pathogenesis and need to be considered during the development of a universal $\operatorname{HIV}$ cure $(6,12)$.

The body's immune system is built around the activities of CD4+ $\mathrm{T}$ cells (13). CD4+ T cells are a significant immune cell subset, which coordinates the immune system and also aid diverse immune $\left(\mathrm{B}, \mathrm{CD} 8^{+} \mathrm{T}\right.$, and $\left.\mathrm{NK}\right)$ cell function (14-16). In addition, they are crucial in generating prompt and protective memory responses against recalled pathogens $(17,18)$. CD4+ T cells are highly plastic and exist as numerous phenotypes like T helper (Th) 1, Th 2, and Th 17, cytotoxic (CD4+ CTL), T follicular helper (Tfh), and $\mathrm{T}$ regulatory ( $\mathrm{T}$ regs) (19). These diverse CD4+ T cell phenotypes express different transcriptional profiles during health and disease, have distinct fates, and carry various functions, including immune regulation ( $C D+\mathrm{T}$ regs) (20). Beyond the simplistic provision of help, CD4+ T cells have also been shown to play other roles like regulation of immune responses $(\mathrm{CD} 4+\mathrm{T}$ regs). They could also directly target infected cells through CD4+ CTLs (13). In supercentenarians, it was suggested that CD4+ CTLs were crucial in healthy aging, where they were found to be expanded while offering long-lasting protection (21).

The hallmark of HIV infection is the progressive loss of total CD4+ T cells and dysregulation of homeostasis, later culminating in acquired immune deficiency syndrome (AIDS) $(22,23)$. Early during infection, HIV causes massive gut damage accompanied by leakage of microbial biproducts into the periphery $(24,25)$. Synchronously, gut dysbiosis occurs as the landscape of the gut microbiome shifts towards more proinflammatory and pathogenic bacterial communities such as Prevotella and Enterobacteriaceae (26-28). As a result of microbial translocation and gut dysbiosis, chronic immune activation and systemic inflammation later ensue $(29,30)$.

Progressively, enhanced CD4+ T cell activation leads to immense CD4+ T cell loss by apoptosis and exaggerated viral cytolysis $(31,32)$. Following combined antiretroviral therapy (cART) initiation, the recovery of absolute CD4+ T-cell counts is often viewed as the benchmark for the immune system reconstitution $(33,34)$. However, ongoing dysregulation of cellular function limits the implementation of robust antiviral responses (35). Furthermore, specific CD4+ T-cell lineages like Tfh have been extensively reported to harbor viral reservoirs within the periphery and lymphoid tissues $(36,37)$. Although CD4+ $\mathrm{T}$ cells are targeted during virus replication and persistence, considerable evidence supports a crucial role of cytotoxic CD4+ T-cell phenotypes in HIV control and slowing disease progression $(38,39)$. During acute HIV infection, a robust HIV-specific CD4+CTL response comprised of elevated granzyme A, interferon-gamma (IFN $\gamma+$ ), and CD40 ligand (CD40L) has been documented to lower viral load set points (40). Johnson et al. also made similar observations and noticed that CD4+ CTLs predominantly expressed perforin, granzyme B, and Eomes during acute HIV infection (38). Subsequently, HIV Nef-specific CD4+ CTLS has been documented to suppress viral replication in macrophages ex-vivo (41).

The lack of a specific CD4+ CTL surface biomarker with consensus across different animal models limits the proper follow-up and interrogation of these cells. Recently, studies by Johnson et al., and Phetsouphanh et al., together used CD57 to identify and track this cell phenotype in different HIV-1 infection conditions $(38,44)$. Despite this, the lack of crossreactive antibodies raised against the CD57 carbohydrate epitope in non-human primate (NHP) models limits its use $(45,46)$. However, recently, in a pre-print article, Nicolet et al. suggested that CD29 enriches human CD4+ CTLs (47). Nonetheless, the utility of this biomarker as a surface marker for CD4+ CTLs remains to be evaluated in NHP models. Although various efforts have been undertaken to explore the effects of CD4+ CTL with HIV disease progression $(39,48)$, the impact of virus-specific functionality of this cell phenotype on the size of replicationcompetent reservoirs remains to be studied. Moreover, the modulation of this cell lineage following exposure to substance abuse such as morphine remains unevaluated.

To address some of above-mentioned questions, we utilized samples obtained from SIV-infected rhesus macaques that were exposed to either morphine or saline. Of these animals, a subset was also treated with cART to suppress viral replication. We found that enhanced CD29+ expression on the surface of CD4+ T cells could be used to identify CD4+ CTLs. Thus, CD29hi CD4+ T cells express higher cytotoxic molecules like CD107a and granzyme $\mathrm{B}$ plus proinflammatory cytokines like IFN- $\gamma$ and TNF- $\alpha$ compared to their CD29lo counterparts. In addition, increased gagspecific secretion of cytokines like IL-21 and granzyme B was accompanied by smaller sizes of replication-competent viral reservoirs suggesting that CD4+ CTLs contribute towards limiting SIV persistence in cART-treated rhesus macaques.

\section{MATERIALS AND METHODS}

\section{Ethics Statement}

This study was approved by the University of Nebraska Medical Center (UNMC) Institutional Animal Care and Use Committee (IACUC) as designated by assigned protocol numbers. For this study, we used: (1) 16-073-07-FC named "The Effect of cART and Drugs of Abuse and the Establishment of CNS Viral Reservoirs". (2) 15-113-01-FC referred to as "The Combinatorial effects of Opiates and Promoter Variant Strains of HIV-1 subtype C on Neuropathogenesis and Latency".

\section{Rhesus Macaques Used for This Study}

For protocol 16-073-07-FC, 6 animals were escalated to $6 \mathrm{mg} / \mathrm{kg}$ twice daily injections of morphine alongside five rhesus macaques 
exposed to saline (controls). Following this, all the animals were infected with $200 \mathrm{TCID}_{50}$ of SIVmac251 and later treated with a cART regimen comprising of $40 \mathrm{mg} / \mathrm{ml}$ emtricitabine (FTC), 20 $\mathrm{mg} / \mathrm{ml}$ tenofovir (TFV), and $2.5 \mathrm{mg} / \mathrm{ml}$ dolutegravir (DTG). Alternatively, protocol 15-113-01-FC involved 8 rhesus macaques separately exposed to equivalent doses of either morphine $(n=4)$ or saline $(n=4)$. Similarly, this was followed with SIVmac251infection at an equivalent dose. However, no subsequent cART was administered. All animal characteristics and treatment regimen details were previously described (10). The experimental schema utilized for the study included a total of 19 rhesus macaques. Briefly, in the untreated group of eight animals, $6-\mathrm{mg} / \mathrm{kg}$ intramuscular (i.m.) injections of morphine were administered twice daily for 2 weeks to four rhesus macaques. Then, continued morphine dosing was maintained for 7 weeks while the remaining four animals received normal saline (control group). After 9 weeks, $200 \mathrm{TCID}_{50}$ of SIVmac251 was intravenously administered to the rhesus macaques and continued with morphine/saline treatments until the end of the study. (B) In the separate group of 11 animals exposed to cART, six were given intramuscular morphine for a total of 9 weeks while five received equal doses of saline (control group). After 9 weeks, 200 TCID $_{50}$ of SIVmac251 was intravenously administered to all animals. Five weeks later, cART was initiated and given daily at $1 \mathrm{ml} / \mathrm{kg}$ body weight up to the termination end of the study. Saline was given to the controls at a similar dose. Lastly, to evaluate the effects of cART on CD29hi CD4+ T cells, we utilized eight rhesus macaques exposed to either four drugs (FTC, tenofovir alafenamide (TAF), DTG and maraviroc (MVC); $\mathrm{n}=4$ ) or two drugs (DTG and FTC; $n=4$ ) that were treated four weeks post SIV inoculation (protocol \#16-001-02-FC, Assessment of Antiretroviral Pharmacology in Lymphoid Tissues using the SIV Macaque Model).

\section{Isolation and Cryopreservation of PBMCs}

Femoral blood, collected in K2-EDTA vacutainer tubes (BD, 367841), was layered above Lymphoprep ${ }^{\text {TM }}$ Density Gradient Medium from STEMCELL Technologies following plasma separation. PBMCs were then later isolated using the density gradient centrifugal separation method described previously (49).

\section{Phenotype Analysis of Immune Cells (Flow Cytometry)}

Cryopreserved PBMCs processed from the blood of rhesus macaques included in this study were utilized for flow cytometry. Post-thaw viabilities of greater than $80 \%$ were considered for further experimentation. Briefly, this involved a brief $12-18 \mathrm{~h}$ rest, and surface staining protocols proceeded at this step. First, Zombie Aqua fixable viability dye was added to discriminate dead cells. After that, all surface markers included in the lineage and integrin/ chemokine receptor panels (Supplementary Table 1) were added at previously titrated volumes. Fixation was then performed using $2 \%$ PFA and samples acquired using the Becton Dickson Fortessa X450 flow cytometer. Intracellular staining protocols involved stimulation of SIV mac239 gag peptide mix spanning 15-mers with 11-aa overlap (ARP-6204) obtained through the NIH HIV Reagent Program, Division of AIDS, NIAID, NIH: Peptide Array,
Simian Immunodeficiency Virus (SIV)mac239 Gag Protein, ARP-6204, contributed by DAIDS/NIAID) and PMA (Phorbol myristate acetate)/ionomycin $(20 \mathrm{ng} / \mathrm{ml}$ and $0.5 \mu \mathrm{g} / \mathrm{ml})$ respectively dissolved in $10 \%$ complete media. Simultaneously, Golgi plug containing Brefeldin A and the Golgi transport inhibitor monensin was added to inhibit the release of cytokines and cytotoxic granules into the supernatant. After 6 hours of stimulation, $20 \mathrm{mM}$ EDTA solution was added. After that, live/ dead exclusion was performed, and surface staining for CD29 PercpCy5.5 was carried out. For the cytotoxicity panel (Supplementary Table 1) involving Eomes and T-bet transcription factors, the fix/perm solution (Tonbo Biosciences) was used for fixation and permeabilization. The remaining antibody cocktail was dissolved in FoxP3 transcription factor buffer (Tonbo biosciences) and added following permeabilization. Alternatively, in the cytokine secretion panel (Supplementary Table 1), 2\% PFA was added for fixation, followed by permeabilization using BD perm wash buffer. Then, cells were incubated with a previously prepared cocktail of antibodies. Following incubation and later washes, the acquisition was performed on the Becton Dickson Fortessa X450 flow cytometer, and analysis was carried out using Flowjo version 10.6 (Trees Star Inc., Ashland, Oregon, USA). For ICS quantification, all values were reported following background subtraction.

\section{Quantification of Plasma Viral Load in Peripheral Blood}

Quantitative real-time PCR (qRT-PCR) was utilized to estimate levels of SIV RNA in EDTA plasma (50). Briefly, RNA was extracted from EDTA plasma using a QIA amp viral RNA mini kit (Qiagen, Germantown, MD, USA; Cat no: 52906). Additional information on primers and probes with details of PCR conditions were described previously (10).

\section{Quantitative Viral Outgrowth Assay (QVOA)}

CD4+ T cells were enriched from PBMCs using the non-human primate microbead CD4+ T cell isolation kit, (STEMCELL Technologies Inc, Seattle, WA, USA) and later resuspended in complete media (RPMI1640, 10\% FBS, 2 mM glutamine, Penstrep (100 U/mL penicillin and $100 \mu \mathrm{g} / \mathrm{mL}$ streptomycin)), $10 \mathrm{U} / \mathrm{mL}$ IL-2 and $300 \mathrm{nM}$ efavirenz (EFV; NIH AIDS Reagent Program). The resultant purified $\mathrm{CD} 4+\mathrm{T}$ cells were then co-stimulated with CD3/CD28 beads (Dynabeads, Life Technologies, Waltham, MA, USA) and 10-fold limiting dilutions ranging from $10^{2}$ to $10^{5}$ cells $/ \mathrm{mL}$ were generated. Following $72 \mathrm{~h}$ of incubation, $10^{5}$ CEMx174 cells were added to aid in viral expansion. This time point was noted as day 0 of incubation and was later prospectively followed for 21 days. SIV RNA measures were then performed using qRT-PCR. Levels of replication-competent viruses were denoted as infectious units per million (IUPM) and were estimated using the IUPMStats v1.0 infection frequency calculator $(10,51)$.

\section{Statistical Analysis}

Prism V9.0 (GraphPad Software) was used for Spearman's rank correlation and paired non-parametric tests with the aid of the Wilcoxon test. In addition, comparisons between morphine and 
saline groups were also performed using the Mann Whitney $\mathrm{U}$ test. Using $\mathrm{R}$ version 3.4.3, a correlation matrix was generated following multiple correlations. Resultant $\mathrm{P}$ values were then corrected for type 1 error with the aid of Holm's correction. Lastly, following Boolean gating in FlowJo, combinatorial expression of multiple cytokines was evaluated and graphed using SPICE ("Simplified Presentation of Incredibly Complex Evaluations”) (52).

\section{RESULTS}

\section{Loss of CD29hi CD4+ T Cells Is Positively Interrelated to Declining Immune Status and Increasing Viral Loads During SIV Infection}

To gain insight into cellular and virological interactions of the peripheral immune system, changes in CD29hi CD4+ T cells alongside viral load and various immune cell subsets during baseline, acute (Day 14), and chronic (Day 245) phases of SIV disease progression (Supplementary Figure 1). Following SIV infection, viral load levels peaked during the acute phase and were maintained through the chronic time point (Figure 1A). Compared to baseline, there was a progressive loss in \%CD4+ $\mathrm{T}$ cell frequencies across the acute phase of infection $(\mathrm{P}=0.0078)$ and the latter chronic phase of infection $(\mathrm{P}=0.0003)$ (Figure 1B). Subsequently, this was accompanied by an expansion of CD8+ T cells during both the acute $(\mathrm{P}=0.0078)$ and chronic $(\mathrm{P}=0.0002)$ phases of untreated SIV infection (Figure 1C). Unsurprisingly, there was a progressive loss in the CD4/CD8 ratio across the acute $(\mathrm{P}=0.0156)$ and chronic $(\mathrm{P}=0.0156)$ phases of infection (Figure 1D). The frequencies of \%CD29hi CD4+ T cells were reduced during the acute $(P=0.0469)$ and chronic $(P=0.0078)$ phases of untreated infection (Figure 1E). Similar trends were observed with absolute CD29hi CD4+ T cells (Supplementary Figure 2A) and absolute CD4+ $\mathrm{T}$ cells (Supplementary Figure 2B), while absolute CD8 $+\mathrm{T}$ cells were elevated during SIV progression (Supplementary Figure 2C). Remarkably, \% CD8+/CD29+ T cells remained stabilized during the acute and chronic phases of untreated SIV infection despite the concurrent expansion of CD8+ T cells (Figure 1F). The percent of both CD29hi CD4+ T cells and CD29+/CD8+ T cells exhibited an increase in activation shown by increased expression of HLA-DR $(\mathrm{P}<0.05)$ (Figures 1G, H). No changes were observed in innatelike CD8+ T cells that express CD16 (Figure 1I). A progressive non-significant reduction of NK cells occurs through the acute to chronic phases of untreated SIV infection (Figure 1J). However, NK cell subset analysis revealed that there was a reduction in \% CD16+CD56- NK cells, $(\mathrm{P}=0.024)$ (Figure 1K) together with concurrent expansions of \%CD16-CD56+ NK cells, $(\mathrm{P}=0.039)$ (Figure 1L) and \%CD16-CD56- NK cells $(\mathrm{P}=0.039)$ that are later maintained during chronic infection (Figure 1M). Multiple Spearman rank correlation analysis revealed that reduced \% CD29hi CD4+ T cells were positively associated with expanding \%CD8+ $\mathrm{T}$ cells ( $\mathrm{rho}=-0.532, \mathrm{P}=0.0044$ ), CD16CD56+ NK cells (rho $=-0.647, \mathrm{P}=0.0006)$ while a positive correlation was seen with improved immune status (CD4/CD8 ratio) ( $r h o=0.42, \mathrm{P}=0.044)$. Additionally, negative associations were noticed with CD16-CD56- NK cells (rho $=-0.7278374)$ and CD16-CD56+ NK cells ( rho $=-0.3928776),($ all $\mathrm{P}<0.05$ ) (Figure 1N). Furthermore, CD16+CD56- NK cells were positively correlated with absolute CD29hi CD4+ T cells (rho = 0.7510 and $\mathrm{P}=0.00001$ ) and absolute CD4+ T cells ( rho $=0.7206$ and $\mathrm{P}=0.0002$ ) respectively (Supplementary Figures 2D, G). In turn, CD16-CD56+ NK cells were associated with loss of CD29 hi CD4+ T cells (Supplementary Figure 2E) and declining absolute CD4+ $\mathrm{T}$ cells (Supplementary Figure $\mathbf{2 H}$ ) during SIV disease progression. Similarly, CD16-CD56- NK cells were also associated with loss of CD29hi CD4+ T cells (Supplementary Figure 2F) and reduced absolute CD4+ T cells (Supplementary Figure 2I) during infection. Lastly, percent and absolute CD29hi CD4+ T cells were seen to negatively associate with viral loads $[($ rho $=-0.57$ and $\mathrm{P}=$ 0.0037 ) and (rho $=-0.7136$ and $\mathrm{P}=0.0001)]$ (Figures 1O, P).

\section{Early Initiation of CART (4 Weeks Post- Infection) Supports Percent CD29hi CD4+ T Cells Restoration and Rescued Polyfunctionality Within the Periphery}

In a separate set of rhesus macaques $(n=8)$, early cART was initiated to test whether depleted peripheral CD29hi CD4+ T cells could be reconstituted. In addition, retrospective time course evaluations were carried out to evaluate how different stages of SIV disease progression and therapy affect the functionality of this cell subset. Representative gating of CD29hi CD4+ T cell subset reveals CD95+, IFN- $\gamma+$ and TNF $\alpha+$ cytokine secretion following PMA/ionomycin stimulation (Figures $\mathbf{2 A}$ and Supplementary Figure 3). Changes in \%CD29hi CD4+ T cells, \%CD95 CD29hi CD4+ T cells, IFN- $\gamma+$ CD29hi CD4+ T cells and TNF- $\alpha+$ CD29hi CD4+ T cells showed that six weeks post untreated SIV infection, a progressive loss CD29hi CD4+ T cells in conjunction with inflammatory cytokines occurred. CD95+ cell expression was elevated during acute SIV infection and remained steadily maintained post-therapy. In contrast, IFN- $\gamma+$ and TNF- $\alpha+$ cytokine levels diminished before ART but recovered after therapy (Figures 2B, E). Finally, early initiation of cART restored lost polyfunctionality of CD29hi CD4+ T cells (\% $(\mathrm{CD} 95+$, IFN- $\gamma+$ and TNF $\alpha+$ ) and [\% CD95+, IFN- $\gamma-$ and TNF $\alpha+)$ ], (Figures 2F).

\section{CD29hi CD4+ T Cells Express Elevated Levels of Pro-Inflammatory and Cytolytic Mediators in Rhesus Macaques Exposed to cART and Morphine During Chronic Infection}

Upon noticing that CD29hi CTLs were depleted during progressive untreated SIV infection, we next turned our efforts to characterizing these cells. Utilizing PBMCs collected from cART treated rhesus macaques, we assessed the extent to which CD4+ CD29hi T cells express diverse integrins (CD11a and $\mathrm{CD} 11 \mathrm{~b}$ ) and the chemokine receptor CX3CR1 (Supplementary Figure 4). In addition, after PMA/ionomycin stimulation, we assessed transcription factors $\mathrm{T}$ bet and Eomes, the cytotoxic 
A

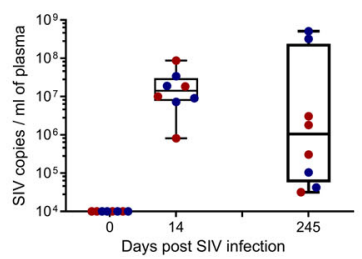

E

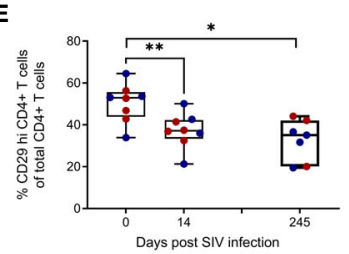

I

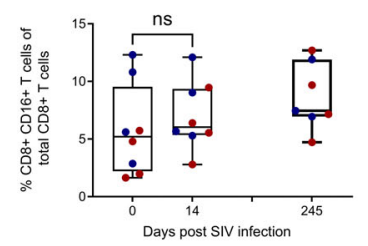

M

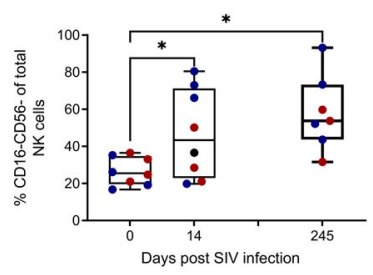

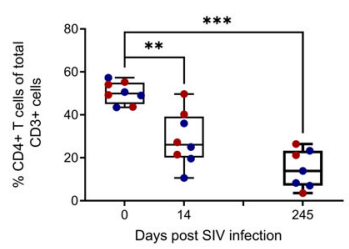

$\mathbf{F}$

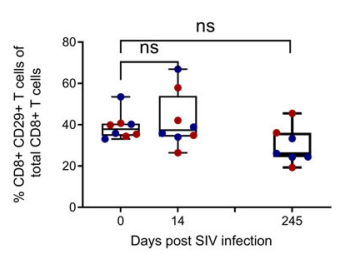

J

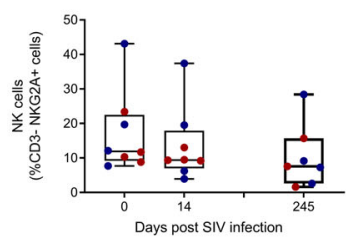

$\mathbf{N}$

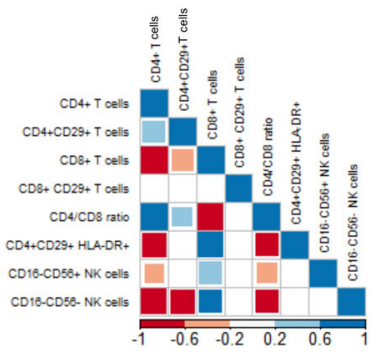

C

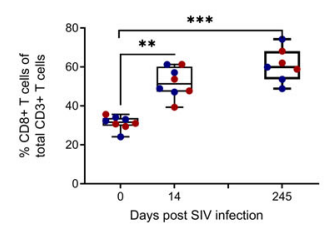

G

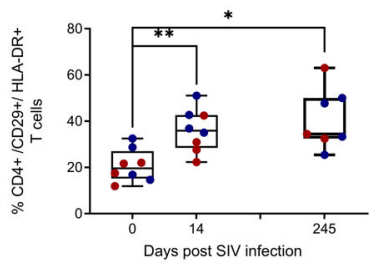

K

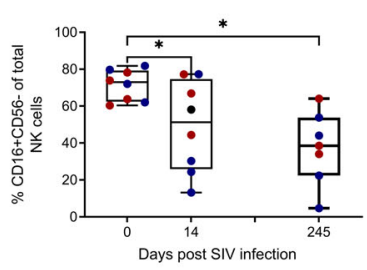

o

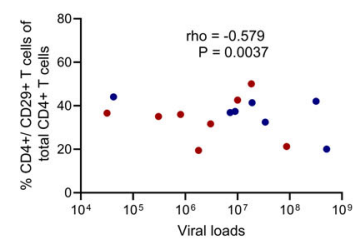

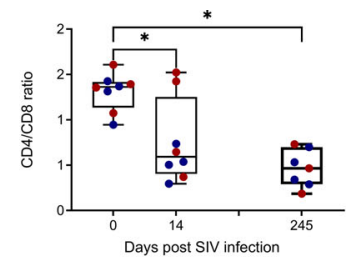

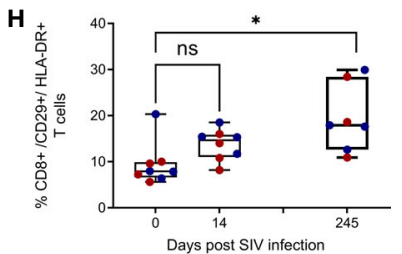

L

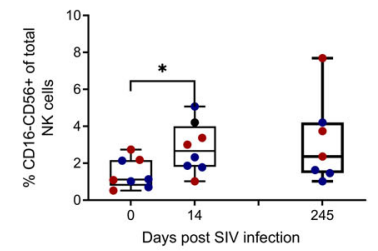

$\mathbf{P}$

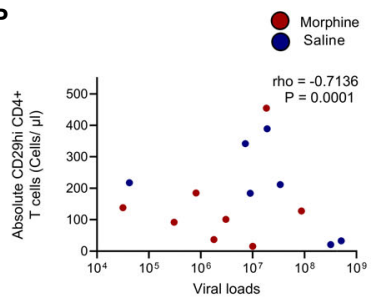

FIGURE 1 | Loss of CD29 hi CD4+ T cells are associated with increased viral loads, declining immune status, and dysregulated NK cell subsets in SIV infected rhesus macaques exposed to either morphine or saline. Viral loads at baseline (day 0), acute (day 14), and chronic (day 245) during untreated SIV infection (A). Changes in percent CD4+ T cells (B), percent CD8+ T cells (C), CD4/CD8 ratio (D), percent CD29+ CD4+ T cells (E), percent CD29+ CD8+ T cells (F), percent CD29+/CD4+/ HLA-DR+ (G), percent CD29+/CD8+/HLA-DR+ (H) and CD8+/CD16+ T cells (I) at the three studied time points (day 0, day 14 and day 245) post infection. Evaluating NK cells and comprising subsets that include changes in total NK cells (J), CD16+CD56- NK cells (K), CD16-CD56+ NK cells (L), and CD16-CD56- NK cells (M) at day 0 , day 14, and day 245 post infection. (N) Correlogram showing correlations between various immune cell subsets and percent CD29+ CD4+ T cells.. Relationship between either (0) percent CD29+ CD4+ T cells or (P) absolute CD29+ CD4+ T cells and viral load. * shows $p<0.05,{ }^{* *}$ indicates $p<0.01$ while ${ }^{* \star *}$ denotes $p<0.001$ resulting from Wilcoxon matched pairs signed rank tests between paired groups of the different comparisons. ns represents non significant. Per visit comparisons between morphine and saline were also performed using the Mann Whitney $U$ test (non- significant).

mediators granzyme B and CD107a, IL-21, T helper (Th) 1 cytokines TNF- $\alpha$ and IFN- $\gamma$ plus the Th2 cytokine IL-4 (Supplementary Figure 5). Surprisingly, there were no significant differences between CD1la expression in CD29hi vs. CD29lo CD4+ T cell subsets (Figure 3A). However, we noticed a three-fold increment of surface CD11b expression on CD29hi versus CD29lo CD4+ $\mathrm{T}$ cells $(\mathrm{P}=0.0020)$ (Figure 3B). Similarly, we observed a close to two-fold elevation in the expression of CX3CR1 in CD29hi CD4+ T cells $(\mathrm{P}=0.0010)$ (Figure 3C). Unexpectedly, the CD29hi CD4+ T cell subset had lower levels of Eomes $(\mathrm{P}=0.0020)$ (Figure 3D) while expressing over two-fold levels of $\mathrm{T}$ bet in comparison to the CD29lo CD4+ $\mathrm{T}$ cell subset $(\mathrm{P}=0.0020)$ (Figure $3 \mathrm{E})$. Moreover, elevated granzyme B expression was observed in the CD29hi CD4+ T cell subset versus the CD29lo CD4+ $\mathrm{T}$ cell subset (Figure 3F). Similarly, the level of granzyme $\mathrm{B}+$ hi expression was elevated in the CD29hi CD4+ T cell subset $(P=0.0010)$ (Figure 3G), which remained augmented even after testing for co-expression of granzyme $\mathrm{B}$ hi and Tbet+ $(\mathrm{P}=0.0010)$ (Figure $\mathbf{3 H})$. The level of IL-4 was similar between CD29hi vs CD29lo CD4+ T cells (Figure 3I) whilst elevated expression of diverse cytokines such as: $\mathrm{CD} 107 \mathrm{a}(\mathrm{P}=0.0010)$ (Figure 3J), IL-21 $(\mathrm{P}=0.0010)$ (Figure 3K), IFN- $\gamma(\mathrm{P}=0.0010)$ (Figure 3L) and TNF- $\alpha(\mathrm{P}=$ 0.0010) (Figure $3 \mathbf{M}$ ) were observed. Similarly, there were higher frequencies of \% IFN- $\gamma+$ and TNF- $\alpha+$ co-expressing CD29hi CD4+ T cells in comparison to CD29lo CD4+ T cells $(\mathrm{P}=0.0087)$ 


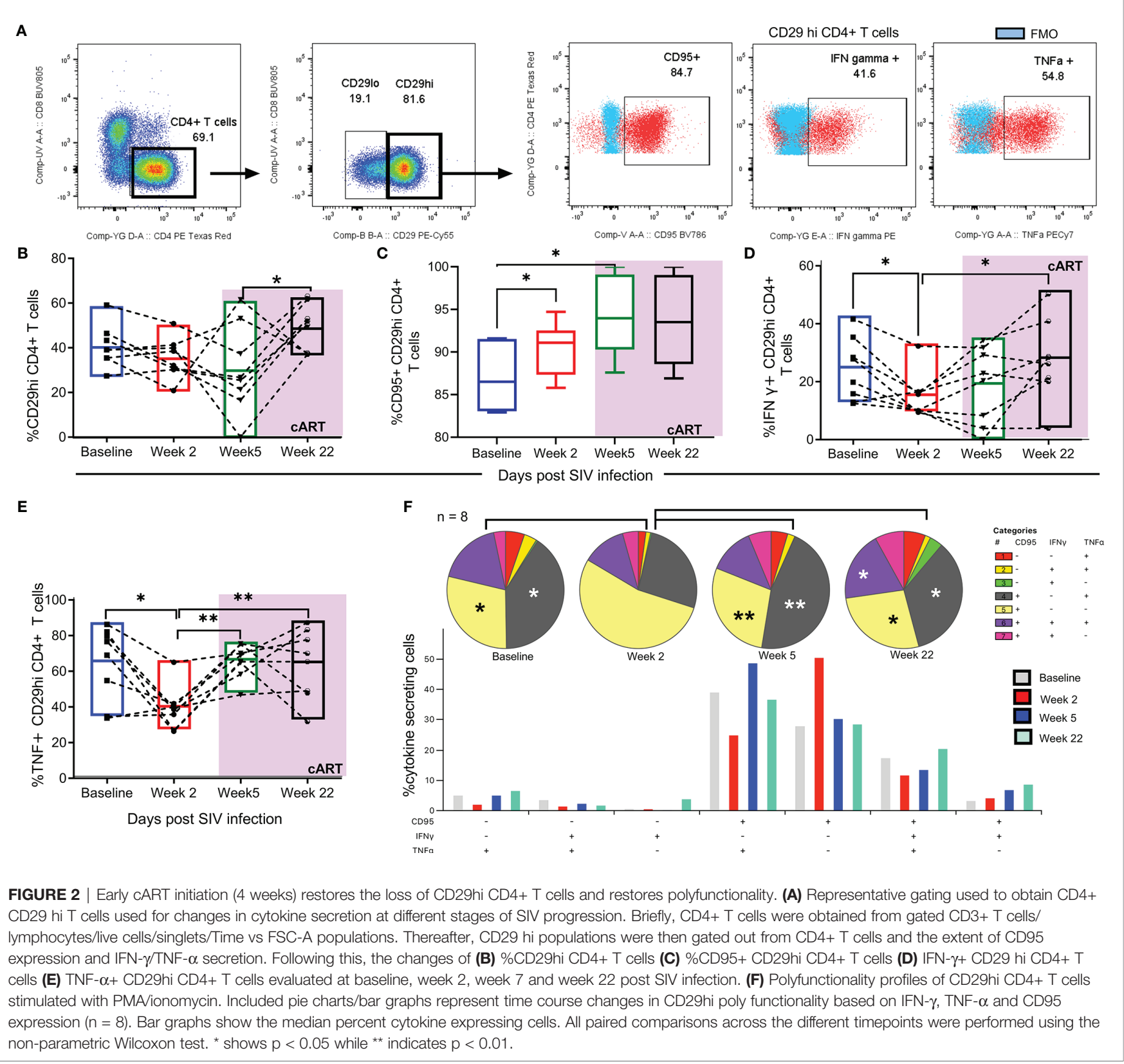

(Figure 3N). Likewise, IL-21+ and IFN $\gamma+$ co-expressing cells are elevated in CD29hi CD4+ T cells in comparison to CD29lo CD4+ T cells $(P=0.0010)$ (Figure 30). Next, we observed that morphine causes downregulation of cytokine secretion ranging from IL-21, TNF- $\alpha$, IFN- $\gamma$, IFN- $\gamma$, and TNF- $\alpha$ dual positive plus IL-21 and IFN$\gamma$ co-expressing CD29hi CD4+ T cells (all $\mathrm{P}<0.01$ ) (Figures 3P-U). Morphine treatment was also observed to similarly downmodulate the expression of inflammatory cytokines and cytotoxic molecules in the CD29lo CD4+ T cell subset (Supplementary Figure 6). By evaluating polyfunctionality of CD29hi CD4+ T cells revealed that the majority of cytokine-producing cells were mainly TNF- $\alpha+$ cells and demonstrated greater frequencies of multiple cytokines secreting cells such as combinations with TNF- $\alpha+$ and CD107a cells, TNF- $\alpha+$ and IL-21 and TNF- $\alpha+$ and IFN- $\gamma+$ co-expressing cells, (all $\mathrm{P}<0.05)$ (Figure 3V).

\section{CD29hi Gag Specific CD4+ T Cells Also Express Elevated Levels of Pro- Inflammatory and Cytolytic Mediators in Rhesus Macaques Exposed to cART and Morphine}

In synchrony with earlier observations seen in Figure 2, CD29 hi CD4+ $\mathrm{T}$ cells expressed higher levels of IFN- $\gamma$ (Figure 4A), CD107a (Figure 4B), IL-21 (Figure 4C), TNF- $\alpha$ (Figure 4D) $($ all $\mathrm{P}<0.05)$ (Supplementary Figure 6). The same trend was seen 


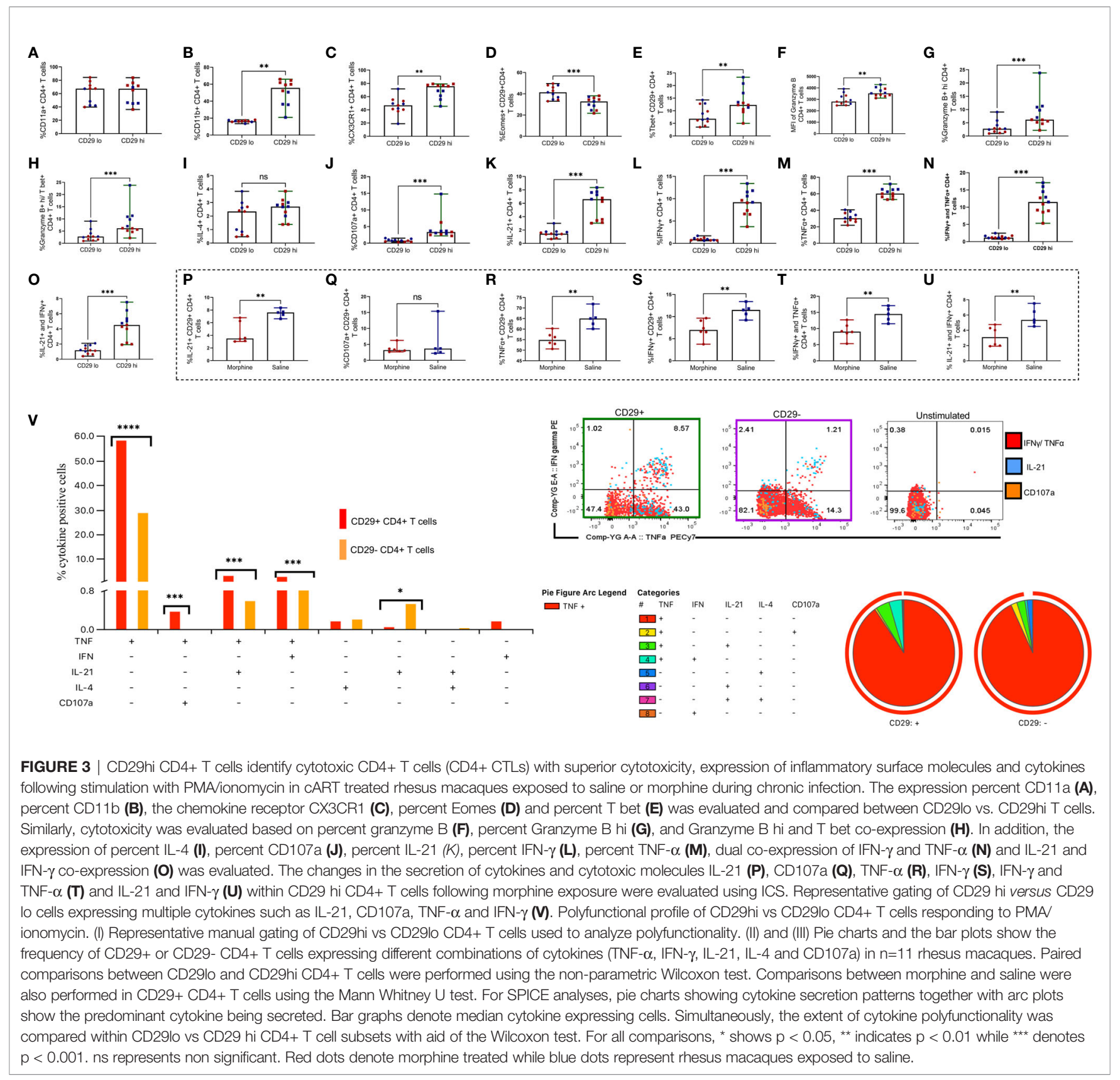

with TNF- $\alpha+$ and CD107a- co-expressing cells (Figure 4F), IFN- $\gamma-$, TNF- $\alpha+$ and CD107a+ co-expressing cells (Figure 4G), percent and MFI granzyme B hi cytolytic molecules (Figures $\mathbf{4 H}$, I) and granzyme B hi and T Bet+ co-expressing cells (Figure 4J) in comparison to CD29lo CD4+ T cells, (all $\mathrm{P}<0.05)$. Notably, no significant differences were observed with levels of IL-4 (Figure 4E), $(\mathrm{P}>0.05)$. In congruence with earlier observations noticed, morphine similarly led to downregulation of various gag-specific cytokines such as IL-21, TNF- $\alpha$, and granzyme B hi T Bet+ in CD29hi CD4+ T cells (all $\mathrm{P}<0.05$ ) (Figures 4K-O). Next, we investigated whether there was a relationship between either IL-21 or granzyme B hi T Bet+ gag specific release on CD29hi CD4+ $\mathrm{T}$ cells with the size of the replication-competent viral reservoir as measured by quantitative viral outgrowth assay (QVOA) (Figure 4P). Negative correlations were observed between QVOA and either IL-21 gag specific CD4+/CD29+T cells or granzyme B hi T bet+ gag specific CD29 hi CD4+ T cells. The association between granzyme B hi T Bet+ CD29hi CD4+ (Figures 4Q, R).

\section{DISCUSSION}

CD4+ CTLs are crucial in limiting the pathogenesis and mediating protection against HIV/SIV infection. It was previously documented that elite controllers, a unique group of 


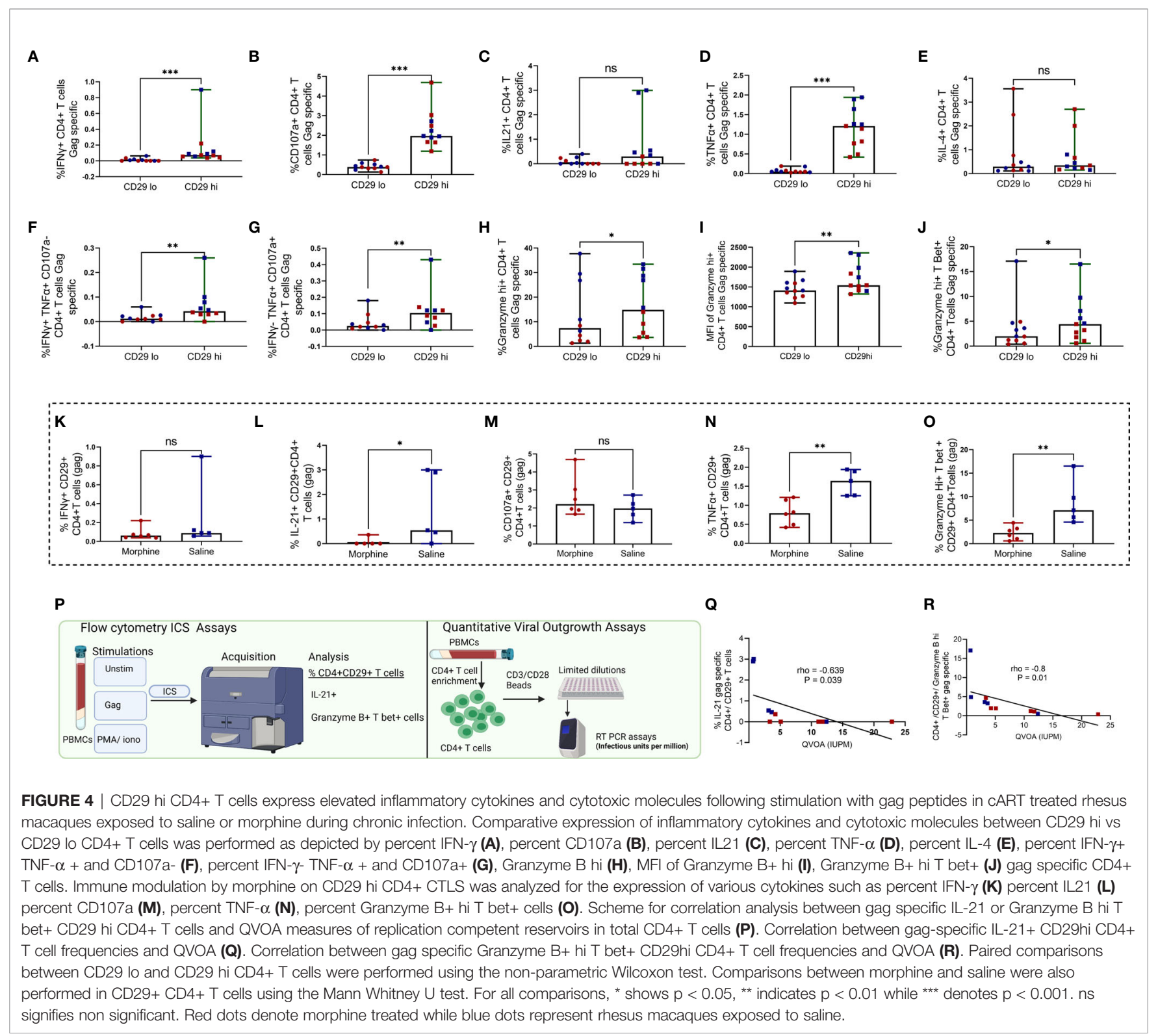

individuals capable of suppressing HIV without therapy, possess greater frequencies of circulating virus-specific CD4+ CTLs (44). Remarkedly, the development of CD4+ CTL escape SIV mutants is accompanied by loss of elite control in rhesus macaques (53). Thus, there is no surprise that declining immune status occurred simultaneously with the loss of proposed CD4+ CTLs and increased viral loads suggesting this cell subset is crucial in limiting HIV pathogenesis.

Similarly, the loss of HIV-specific CD4+ CTL function has been reported in chronic progressors (44). Predictably, during progressive infection, CD29hi CD4+ T cells exhibited increased HLA-DR activation levels, hinting that this cell phenotype could be targeted and depleted by the virus (54). Furthermore, the expansion of cytokine secreting (CD16-CD56+) and double negative CD16-CD56- NK cells are crucial for limiting viremia post-peak viral loads $(55,56)$. However, the loss of CD16 expression on prevailing $\mathrm{NK}$ cell subsets followed by the decrease of both CD29hi CD4+ T cells and absolute CD4+ T cells further highlights SIV-induced NK cell and CD4+ CTL dysfunction during disease progression (57).

During our time course studies, our observation that timely initiation of therapy reconstitutes peripheral CD29hi CD4+ T cells and restores IFN- $\gamma$, TNF- $\alpha$, and CD95 polyfunctionality, in turn, supports findings from other groups who have reported that early ART preserves T cell function $(58,59)$. Following gag and PMA/ ionomycin stimulation, CD29hi CD4+ T cells display cytotoxic properties through elevated secretion of proinflammatory cytokines such as IFN- $\gamma$, TNF- $\alpha$, and IL-21 or cytolytic molecules such as granzyme and CD107a (60). In turn, increased expression of $\mathrm{T}$ bet dually favors IFN- $\gamma$ and TNF- $\alpha$ 
secretion while supporting cytotoxicity by binding to downstream promoters of granzyme B and perforin genes (61).

Similarly, augmented expression of CX3CR1 also tends to favor CD4+ CTL transmigration into inflamed tissue, as exemplified in Dengue, where virus-specific CX3CR1 CD4+CTLs mediate protective cytotoxicity (62). Similarly, Chen et al. showed that a cytomegalovirus-driven CD57hi CD4+ CTL phenotype uses CX3CR1 to facilitate vascular trafficking by attaching to the fractalkine receptor on the endothelium during HIV infection (63).

Since CD29hi CD4+ T cells exhibit greater polyfunctionality as demonstrated by a distinct functional profile typified by elevated secretion of multiple inflammatory cytokines and cytotoxic molecules, they could serve as robust antiviral mediators that directly target virus-infected cells (59). In agreement with this, we noted that increased ex-vivo secretion of IL-21 in response to gag was negatively associated with the size of the replication-competent viral reservoir within the periphery. In a separate study, IL-21 secretion by CD4+ T cells was noted to support CD8+ cytotoxic T cell responses in chronic progressors, even during the late-stage infection, leading to diminished virus replication (64). Furthermore, therapeutic administration of IL21 has been documented to reduce the size of viral reservoirs in rhesus macaques $(64,65)$.

The association between granzyme B hi T Bet+ CD29hi CD4+ $\mathrm{T}$ cells and diminished replication-competent viral reservoirs mirrors earlier findings by a separate study group found that HIV specific granzyme B and not IFN-g CD8 CTLs were linked to reduced HIV reservoirs during acute HIV infection (42). In addition, increased inflammation, as noted by elevated levels of pro inflammatory cytokines such as IL-18 (data not shown), has been linked to development of AIDS $(43,66)$. The observation that elevated CD29hi CD4+ CTLs were negatively associated with proinflammatory plasma IL-18 during untreated SIV infection, data not shown, corroborates the importance of these cells in fostering better disease outcomes.

The observation that morphine downmodulated the secretion of virus-specific proinflammatory cytokines and the release of granzyme B by CD4+ CD29hi CTLs coincides with several reports that suggest that this drug inhibits $\mathrm{CD} 4+\mathrm{T}$ cell release of Th1 cytokines (IFN $\gamma$, TNF- $\alpha$ ) while causing a shift towards Th2 phenotypes $(67,68)$. However, future studies are needed to evaluate the mechanisms by which morphine causes immune modulation of this cell subset. Further, larger sample sizes and studies using sorted CD29hi CD4+ CTLs are necessary for further validation of the contribution of this cell phenotype towards targeting persistence.

Additional future strategies aimed at improving the CD4+ CTL functionality, such as utilizing chimeric engineering strategies could reinvigorate CD4+ CTLs fostering improved disease outcomes $(69,70)$. Further, the unexpected transcription profile of lower Eomes expression in CD29hi CD4+ $\mathrm{T}$ cells while harnessing augmented levels of Tbet warrants further research in understanding how regulation of transcription factors affects CD4+ CTL functionality and differentiation. In their CD8+ T cell counterparts, Smith et al., revealed that cells expressing Tbet hi and Eomes lo profiles are more efficient in recognizing major histocompatibility complex
(MHC) peptides (71). Finally, there is a need to track changes in this cell lineage in body compartments such as lymph nodes, central nervous system, and the gut that provide safe niches for low-level virus replication, evolution, and persistence following effective cART (72).

In conclusion, we demonstrate that CD29 could be reliably used to identify CD4+ CTLs in rhesus macaques. Furthermore, CD29hi CD4+ CTLs are beneficial towards limiting SIV pathogenesis by limiting virus replication and secreting crucial cytokines associated with reducing the size of the viral reservoir within the periphery.

\section{DATA AVAILABILITY STATEMENT}

The original contributions presented in the study are included in the article/Supplementary Material. Further inquiries can be directed to the corresponding author.

\section{ETHICS STATEMENT}

The animal study was reviewed and approved by University of Nebraska Medical Center (UNMC) Institutional Animal Care and Use Committee (IACUC).

\section{AUTHOR CONTRIBUTIONS}

$\mathrm{OO}$ and SNB designed the conceptual framework of the study and analyzed the data. OO performed all the flow cytometry experiments, wrote the initial draft of the manuscript. SJ, KP, $\mathrm{MT}$, and AA carried out virological assays. SJB, HF, AP, CF, and SNB supported fundraising for the animal studies and edited the manuscript. All authors contributed to the article and approved the submitted version.

\section{FUNDING}

This study was supported by NIH grants R01DA043164, P30MH062261 to SNB, SJB, and HF, R01DA041751 to SNB and SJB, and R01AI124965 to CF. The funders had no role in designing the study or in the interpretation of the results.

\section{ACKNOWLEDGMENTS}

We appreciate the staff and veterinarians of the University of Nebraska Medical Center (UNMC) Comparative Medicine department for housing and animal procedures. We also appreciate other team members like Brenda M. Morsey, Benjamin Lamberty, Shannon Callen, and personnel from Dr. Byrareddy's laboratory who routinely took care of the rhesus macaques included in this study. We are also grateful to members of the flow core section at UNMC. Finally, we thank 
NIH HIV Reagent Program, Division of AIDS, NIAID, NIH for the provision of Peptide Array, Simian Immunodeficiency Virus (SIV) mac239 Gag Protein, ARP-6204.

\section{SUPPLEMENTARY MATERIAL}

The Supplementary Material for this article can be found online at: https://www.frontiersin.org/articles/10.3389/fimmu.2021. 734871/full\#supplementary-material

Supplementary Figure 1 | Longitudinal Changes In Diverse Lymphocyte Subsets During Progressive Untreated Siv Infection. Gating strategy based on a representative sample used for delineation of lymphocyte subpopulations within PBMCs obtained from untreated SIV rhesus macaques. Lymphocytes were gated out based on size and complexity (FSC-a vs. SSC-a). NKG2A vs. CD3+ was used to exclude CD3+ T cells, NKT cells, and NK cells. CD3+ T cells were later differentiated based on CD4+ and CD8+ markers and expression of CD29 was used to mark cytotoxicity in either subset. HLA-DR levels were then used to identify activation levels in either CD4+ or CD8+ T cells. MAIT cells were also studied based on CD161 and $V \alpha 7.2 \mathrm{~J} \alpha$ co-expression. CD16+ CD8+ T cells (innate like CD8+ T cells) were also evaluated. NK cell subsets based on CD16 and CD56 expression were also studied for maturity based on CD27 and activation based on HLA-DR expression.

Supplementary Figure 2 | Relationship between absolute t cell subsets and percent frequnencies of $\mathrm{nk}$ cell subsets during untreated siv disease progression. Changes in absolute: (A) CD29hi CD4+ T cells (B) CD4+ T cells (C) CD8+ T cells during baseline (day 0), day 14 and day 245 past SIV infection. Correlation between absolute CD29hi CD4+ T cells and: (D) \%CD16+CD56- NK cells (E) \%CD16-CD56+ NK cells (F) \%CD16-CD56- NK cells. Additional correlations were also performed that included the association between absolute CD4+ T cells and: (G) \%CD16+CD56- NK cells (H) \%CD16-CD56+ NK cells (I) \%CD16-CD56- NK cells. For (A-C), ${ }^{* *}$ indicates $p<0.001$ while ${ }^{* \star *}$ denotes $p<0.0001$ resulting from Wilcoxon matched pairs signed rank tests between paired groups of the different comparisons. Per visit comparisons between morphine and saline were also performed using the Mann Whitney $U$ test (non- significant).

Supplementary Figure 3 | Representative Gating Of Time Course Changes In Cd29hi Cd4+ T Cells Across The Various Studied Time Points (Baseline, Week 2, Week 7 And Week 22). Representative gating for rhesus macaque CF42 indicating

\section{REFERENCES}

1. Kuehn BM. Study Suggests a Second Patient Has Been Cured of HIV. JAMA (2020) 323:1886. doi: 10.1001/jama.2020.7626

2. Woldemeskel BA, Kwaa AK, Blankson JN. Viral Reservoirs in Elite Controllers of HIV-1 Infection: Implications for HIV Cure Strategies. EBioMedicine (2020) 62:103118. doi: 10.1016/j.ebiom.2020.103118

3. Macedo AB, Novis CL, Bosque A. Targeting Cellular and Tissue HIV Reservoirs With Toll-Like Receptor Agonists. Front Immunol (2019) 10:2450. doi: 10.3389/fimmu.2019.02450

4. Chaillon A, Gianella S, Dellicour S, Rawlings SA, Schlub TE, De Oliveira MF, et al. HIV Persists Throughout Deep Tissues With Repopulation From Multiple Anatomical Sources. J Clin Invest (2020) 130:1699-712. doi: 10.1172/JCI134815

5. Busman-Sahay K, Starke CE, Nekorchuk MD, Estes JD. Eliminating HIV Reservoirs for a Cure: The Issue Is in the Tissue. Curr Opin HIV AIDS (2021) 16:200-8. doi: 10.1097/COH.0000000000000688

6. Hrdy DB. Cultural Practices Contributing to the Transmission of Human Immunodeficiency Virus in Africa. Rev Infect Dis (1987) 9:1109-19. doi: 10.1093/clinids/9.6.1109

7. Chang CC, Crane M, Zhou J, Mina M, Post JJ, Cameron BA, et al. HIV and Co-Infections. Immunol Rev (2013) 254:114-42. doi: 10.1111/imr.12063

8. Degenhardt L, Peacock A, Colledge S, Leung J, Grebely J, Vickerman P, et al. Global Prevalence of Injecting Drug Use and Sociodemographic changes in CD95 expression, IFN- $\gamma$ and TNF- $\alpha$ secretion across CD29hi CD4+ T cells within the studied time points. FMO controls also included for the accurate placement of gates.

Supplementary Figure 4 | Determination Of Integrin (Cd11a And Cd11b) And Chemokine Receptor Expression (Cx3cr1) On Cd29hi Vs Cd29lo Cd4 T Cells During Art Treated Chronic Siv Infection. To achieve this, a time vs FSC-A gate was used to obtain a steady stream of cells during cell acquisition that is devoid of interruptions caused by micro bubbles, clogs or air. The FSC-A vs FSC-H gate and SSC-A vs SSC-H was used to obtain single cells. Dead cells were excluded using positive zombie aqua live dead staining. Lymphocytes were then segregated based on FSC-A vs SSC-A gate based on size and complexity and T cells later gated off using the CD3+ marker. CD4+ T cells were then further gated followed by the extent of their expression of CD29. Comparisons of CD29hi vs CD29lo CD4+ T cells were then carried out to evaluate the expression of CD11a and CD11B integrins together with the chemokine receptor CX3CR1. Fluorescent minus one (FMO) controls were included to set a background for gate placement.

Supplementary Figure 5 | Evaluation Of Cytokine Expression In Cart Treated Siv Infected Macaues Exposed To Morphine Or Saline. Cells were excluded based on the FSC-a vs. time to obtain cells in a single stream. Doublets were excluded using FSC-A vs. FSC-H/SSC-A vs. SSC-H. Live cells were then excluded based on Live Dead Zombie expression. CD3+ T cells were then identified and CD4+ or CD8+ T cells further profiled. CD29 expression in CD4+ T cells was then profiled and various cytokines such as IFN- $\gamma$, CD107a, IL21, TNF- $\alpha$ and IL-4 in CD29 lo vs CD29 hi $\mathrm{CD} 4+\mathrm{T}$ cells unstimulated, gag and PMA/ionomycin conditions.

Supplementary Figure 6 | evaluation of cytokine secretion and cytotoxic molecule expression in cd29lo cd4 + t cells of cart treated siv infected macaques exposed to morphine or saline. Levels of (A) ifn- $\gamma$ (B) il-4 (C) tnf- $\alpha$ (D) cd107a (E) mfi of granzyme b (F) granzyme b hi (G) ifn- $\gamma$ and tnf- $\alpha(\mathbf{H})$ il-21 and ifn- $\gamma$ expression in cd29lo cd4 + t cells.

Supplementary Figure 7 | evaluation of cytotoxic molecules, transcription factors in cart treated siv infected macaues exposed to morphine or saline. Cd3+ $\mathrm{t}$ cells were identified from live cells. Cd4+ t cells were then gated out followed by testing for cd29 expression. In unstimulated, gag or pma/ionomycin conditions, percent expression of: the cytotoxic molecule granzyme together with transcription factors like t bet or eomes were evaluated in cd29 hi vs cd29 lo subsets.

Supplementary Table 1 | various antibody panels used for the study. Exhaustive list of monoclonal antibodies with insights into specificity, fluorochrome tagged, ab clone, vendor/source together with volumes/test used.

Characteristics and Prevalence of HIV, HBV, and HCV in People Who Inject Drugs: A Multistage Systematic Review. Lancet Global Health (2017) 5 e1192-207. doi: 10.1016/S2214-109X(17)30375-3

9. Power J, Westle A, Dowsett GW, Lucke J, Tucker JD, Sugarman J, et al. Perceptions of HIV Cure Research Among People Living With HIV in Australia. PloS One (2018) 13:e0202647. doi: 10.1371/journal.pone.0202647

10. Acharya A, Olwenyi OA, Thurman M, Pandey K, Morsey BM, Lamberty B, et al. Chronic Morphine Administration Differentially Modulates Viral Reservoirs in a Simian Immunodeficiency Virus SIVmac251-Infected Rhesus Macaque Model. J Virol (2021) 95:e01657-01620. doi: 10.1128/ JVI.01657-20

11. Olwenyi OA, Asingura B, Naluyima P, Anywar GU, Nalunga J, Nakabuye M, et al. In-Vitro Immunomodulatory Activity of Azadirachta Indica A.Juss. Ethanol: Water Mixture Against HIV Associated Chronic CD4(+) T-Cell Activation/ Exhaustion. BMC Complement Med Ther (2021) 21:114. doi: 10.1186/s12906-021-03288-0

12. Dybul M, Attoye T, Baptiste S, Cherutich P, Dabis F, Deeks SG, et al. The Case for an HIV Cure and How to Get There. Lancet HIV (2020) 8:e51-8. doi: 10.1016/S2352-3018(20)30232-0

13. Swain SL, Mckinstry KK, Strutt TM. Expanding Roles for CD4 + T Cells in Immunity to Viruses. Nat Rev Immunol (2012) 12:136-48. doi: 10.1038/nri3152

14. Jost S, Tomezsko PJ, Rands K, Toth I, Lichterfeld M, Gandhi RT, et al. CD4+ T-Cell Help Enhances NK Cell Function Following Therapeutic HIV-1 Vaccination. J Virol (2014) 88:8349-54. doi: 10.1128/JVI.00924-14 
15. Ayasoufi K, Fan R, Fairchild RL, Valujskikh A. CD4 T Cell Help via B Cells Is Required for Lymphopenia-Induced CD8 T Cell Proliferation. J Immunol (2016) 196:3180. doi: 10.4049/jimmunol.1501435

16. Laidlaw BJ, Craft JE, Kaech SM. The Multifaceted Role of CD4(+) T Cells in CD8(+) T Cell Memory. Nat Rev Immunol (2016) 16:102-11. doi: 10.1038/ nri.2015.10

17. Gray JI, Westerhof LM, Macleod MK. The Roles of Resident, Central and Effector Memory CD 4 T-Cells in Protective Immunity Following Infection or Vaccination. Immunology (2018) 154:574-81. doi: 10.1111/imm.12929

18. Beura LK, Fares-Frederickson NJ, Steinert EM, Scott MC, Thompson EA, Fraser KA, et al. CD4+ Resident Memory $\mathrm{T}$ Cells Dominate Immunosurveillance and Orchestrate Local Recall Responses. J Exp Med (2019) 216:1214-29. doi: 10.1084/jem.20181365

19. Hoefig KP, Heissmeyer V. Posttranscriptional Regulation of T Helper Cell Fate Decisions. J Cell Biol (2018) 217:2615-31. doi: 10.1083/jcb.201708075

20. Caza T, Landas S. Functional and Phenotypic Plasticity of CD4+ T Cell Subsets. BioMed Res Int (2015) 2015:521957. doi: 10.1155/2015/521957

21. Hashimoto K, Kouno T, Ikawa T, Hayatsu N, Miyajima Y, Yabukami H, et al. Single-Cell Transcriptomics Reveals Expansion of Cytotoxic CD4 T Cells in Supercentenarians. Proc Natl Acad Sci USA (2019) 116:24242-51. doi: 10.1073/pnas.1907883116

22. Olwenyi OA, Naluyima P, Cham F, Quinn TC, Serwadda D, Sewankambo NK, et al. Brief Report: Differential Associations of Interleukin 6 and Intestinal Fatty Acid-Binding Protein With Progressive Untreated HIV-1 Infection in Rakai, Uganda. J acquired Immune deficiency syndromes (1999) (2016) 72:1520. doi: 10.1097/QAI.0000000000000915

23. Vidya Vijayan KK, Karthigeyan KP, Tripathi SP, Hanna LE. Pathophysiology of CD4+ T-Cell Depletion in HIV-1 and HIV-2 Infections. Front Immunol (2017) 8:580. doi: 10.3389/fimmu.2017.00580

24. Estes JD, Harris LD, Klatt NR, Tabb B, Pittaluga S, Paiardini M, et al. Damaged Intestinal Epithelial Integrity Linked to Microbial Translocation in Pathogenic Simian Immunodeficiency Virus Infections. PloS Pathog (2010) 6:e1001052. doi: 10.1371/journal.ppat.1001052

25. Sandler NG, Douek DC. Microbial Translocation in HIV Infection: Causes, Consequences and Treatment Opportunities. Nat Rev Microbiol (2012) 10:655-66. doi: 10.1038/nrmicro2848

26. Dillon SM, Frank DN, Wilson CC. The Gut Microbiome and HIV-1 Pathogenesis: A Two-Way Street. Aids (2016) 30:2737-51. doi: 10.1097/ QAD.0000000000001289

27. Dillon SM, Lee EJ, Kotter CV, Austin GL, Gianella S, Siewe B, et al. Gut Dendritic Cell Activation Links an Altered Colonic Microbiome to Mucosal and Systemic T-Cell Activation in Untreated HIV-1 Infection. Mucosal Immunol (2016) 9:24-37. doi: 10.1038/mi.2015.33

28. Dubourg G, Lagier J-C, Hüe S, Surenaud M, Bachar D, Robert C, et al. Gut Microbiota Associated With HIV Infection Is Significantly Enriched in Bacteria Tolerant to Oxygen. BMJ Open Gastroenterol (2016) 3:e000080. doi: 10.1136/bmigast-2016-000080

29. Klatt NR, Funderburg NT, Brenchley JM. Microbial Translocation, Immune Activation, and HIV Disease. Trends Microbiol (2013) 21:6-13. doi: 10.1016/ j.tim.2012.09.001

30. Dillon SM, Kibbie J, Lee EJ, Guo K, Santiago ML, Austin GL, et al. Low Abundance of Colonic Butyrate-Producing Bacteria in HIV Infection Is Associated With Microbial Translocation and Immune Activation. AIDS (London England) (2017) 31:511-21. doi: 10.1097/QAD.0000000000001366

31. Groux H, Torpier G, Monté D, Mouton Y, Capron A, Ameisen JC. ActivationInduced Death by Apoptosis in CD4+ $\mathrm{T}$ Cells From Human Immunodeficiency Virus-Infected Asymptomatic Individuals. J Exp Med (1992) 175:331-40. doi: 10.1084/jem.175.2.331

32. Doitsh G, Cavrois M, Lassen KG, Zepeda O, Yang Z, Santiago ML, et al. Abortive HIV Infection Mediates CD4 T Cell Depletion and Inflammation in Human Lymphoid Tissue. Cell (2010) 143:789-801. doi: 10.1016/ j.cell.2010.11.001

33. Handoko R, Colby DJ, Kroon E, Sacdalan C, De Souza M, Pinyakorn S, et al. Determinants of Suboptimal CD4(+) T Cell Recovery After Antiretroviral Therapy Initiation in a Prospective Cohort of Acute HIV-1 Infection. $J$ Int AIDS Soc (2020) 23:e25585-5.

34. Hernández-Walias F, Ruiz-De-León MJ, Rosado-Sánchez I, Vázquez E, Leal $\mathrm{M}$, Moreno S, et al. New Signatures of Poor CD4 Cell Recovery After
Suppressive Antiretroviral Therapy in HIV-1-Infected Individuals: Involvement of miR-192, IL-6, Scd14 and miR-144. Sci Rep (2020) 10:2937. doi: $10.1038 / s 41598-020-60073-8$

35. Wilson EMP, Sereti I. Immune Restoration After Antiretroviral Therapy: The Pitfalls of Hasty or Incomplete Repairs. Immunol Rev (2013) 254:343-54. doi: 10.1111/imr.12064

36. Aid M, Dupuy FP, Moysi E, Moir S, Haddad EK, Estes JD, et al. Follicular CD4 T Helper Cells As a Major HIV Reservoir Compartment: A Molecular Perspective. Front Immunol (2018) 9:895. doi: 10.3389/ fimmu.2018.00895

37. Godinho-Santos A, Foxall RB, Antão AV, Tavares B, Ferreira T, SerraCaetano A, et al. Follicular Helper T Cells Are Major Human Immunodeficiency Virus-2 Reservoirs and Support Productive Infection. J Infect Dis (2019) 221:122-6. doi: 10.1093/infdis/jiz431

38. Johnson S, Eller M, Teigler JE, Maloveste SM, Schultz BT, Soghoian DZ, et al. Cooperativity of HIV-Specific Cytolytic CD4 T Cells and CD8 T Cells in Control of HIV Viremia. J Virol (2015) 89:7494-505. doi: 10.1128/JVI.00438-15

39. Sanchez-Martinez A, Perdomo-Celis F, Acevedo-Saenz L, Rugeles MT, Velilla PA. Cytotoxic CD4(+) T-Cells During HIV Infection: Targets or Weapons? J Clin Virol (2019) 119:17-23. doi: 10.1016/j.jcv.2019.08.004

40. Soghoian DZ, Jessen H, Flanders M, Sierra-Davidson K, Cutler S, Pertel T, et al. HIV-Specific Cytolytic CD4 T Cell Responses During Acute HIV Infection Predict Disease Outcome. Sci Trans Med (2012) 4:123ra125123ra125. doi: 10.1126/scitranslmed.3003165

41. Zheng N, Fujiwara M, Ueno T, Oka S, Takiguchi M. Strong Ability of NefSpecific CD4+ Cytotoxic T Cells To Suppress Human Immunodeficiency Virus Type 1 (HIV-1) Replication in HIV-1-Infected CD4+ T Cells and Macrophages. J Virol (2009) 83:7668-77. doi: 10.1128/JVI.00513-09

42. Yue FY, Cohen JC, Ho M, Rahman A, Liu J, Mujib S, et al. HIV-Specific Granzyme B-Secreting But Not Gamma Interferon-Secreting T Cells Are Associated With Reduced Viral Reservoirs in Early HIV Infection. J Virol (2017) 91:e02233-02216. doi: 10.1128/JVI.02233-16

43. Iannello A, Samarani S, Debbeche O, Boulassel MR, Tremblay C, Toma E, et al. Potential Role of IL-18 in the Immunopathogenesis of AIDS, HIVAssociated Lipodystrophy and Related Clinical Conditions. Curr HIV Res (2010) 8:147-64. doi: 10.2174/157016210790442713

44. Phetsouphanh C, Aldridge D, Marchi E, Munier CML, Meyerowitz J, Murray L, et al. Maintenance of Functional CD57+ Cytolytic CD4+ T Cells in HIV+ Elite Controllers. Front Immunol (2019) 10:1844. doi: 10.3389/fimmu. 2019.01844

45. Ram DR, Manickam C, Hueber B, Itell HL, Permar SR, Varner V, et al. Tracking KLRC2 (NKG2C)+ Memory-Like NK Cells in SIV+ and rhCMV+ Rhesus Macaques. PloS Pathog (2018) 14:e1007104. doi: 10.1371/ journal.ppat.1007104

46. Resource, N.H.P.R. (2021). Available at: https://www.nhpreagents.org/ ReactivityDatabase.

47. Nicolet BP, Guislain A, Wolkers MC. CD29 Enriches for Cytotoxic Human CD4+ T Cells. BioRxiv (2021) 2021.2002.2010.430576. doi: 10.1101/ 2021.02.10.430576

48. Porichis F, Kaufmann DE. HIV-Specific CD4 T Cells and Immune Control of Viral Replication. Curr Opin HIV AIDS (2011) 6:174-80. doi: 10.1097/ COH.0b013e3283454058

49. Woollard SM, Olwenyi OA, Dutta D, Dave RS, Mathews S, Gorantla S, et al. Preliminary Studies on Immune Response and Viral Pathogenesis of Zika Virus in Rhesus Macaques. Pathogens (2018) 7:70. doi: 10.3390/ pathogens 7030070

50. Byrareddy SN, Kallam B, Arthos J, Cicala C, Nawaz F, Hiatt J, et al. Targeting $\alpha 4 \beta 7$ Integrin Reduces Mucosal Transmission of Simian Immunodeficiency Virus and Protects Gut-Associated Lymphoid Tissue From Infection. Nat Med (2014) 20:1397-400. doi: 10.1038/nm.3715

51. Olwenyi OA, Acharya A, Routhu NK, Pierzchalski K, Jones JW, Kane MA, et al. Retinoic Acid Improves the Recovery of Replication-Competent Virus From Latent SIV Infected Cells. Cells (2020) 9:2076. doi: 10.3390/cells9092076

52. Roederer M, Nozzi JL, Nason MC. SPICE: Exploration and Analysis of PostCytometric Complex Multivariate Datasets. Cytometry Part A (2011) 79A:167-74. doi: 10.1002/cyto.a.21015

53. Burwitz BJ, Giraldo-Vela JP, Reed J, Newman LP, Bean AT, Nimityongskul FA, et al. CD8+ and CD4+ Cytotoxic T Cell Escape Mutations Precede 
Breakthrough SIVmac239 Viremia in an Elite Controller. Retrovirology (2012) 9:91-1. doi: 10.1186/1742-4690-9-91

54. Veazey RS, Tham IC, Mansfield KG, Demaria M, Forand AE, Shvetz DE, et al. Identifying the Target Cell in Primary Simian Immunodeficiency Virus (SIV) Infection: Highly Activated Memory CD4(+) T Cells Are Rapidly Eliminated in Early SIV Infection In Vivo. J Virol (2000) 74:57-64. doi: 10.1128/ JVI.74.1.57-64.2000

55. Carville A, Evans TI, Reeves RK. Characterization of Circulating Natural Killer Cells in Neotropical Primates. PloS One (2013) 8:e78793. doi: 10.1371/ journal.pone.0078793

56. Hong H, Rajakumar P, Billingsley J, Reeves RK, Johnson R. No Monkey Business: Why Studying NK Cells in Non-Human Primates Pays Off. Front Immunol (2013) 4:32. doi: 10.3389/fimmu.2013.00032

57. Vargas-Inchaustegui DA, Ying O, Demberg T, Robert-Guroff M. Evaluation of Functional NK Cell Responses in Vaccinated and SIV-Infected Rhesus Macaques. Front Immunol (2016) 7:340. doi: 10.3389/fimmu.2016.00340

58. Schuetz A, Deleage C, Sereti I, Rerknimitr R, Phanuphak N, Phuang-Ngern Y, et al. Initiation of ART During Early Acute HIV Infection Preserves Mucosal Th17 Function and Reverses HIV-Related Immune Activation. PloS Pathog (2014) 10:e1004543. doi: 10.1371/journal.ppat.1004543

59. Salido J, Ruiz MJ, Trifone C, Figueroa MI, Caruso MP, Gherardi MM, et al. Phenotype, Polyfunctionality, and Antiviral Activity of In Vitro Stimulated CD8+ T-Cells From HIV+ Subjects Who Initiated cART at Different TimePoints After Acute Infection. Front Immunol (2018) 9:2443. doi: 10.3389/ fimmu.2018.02443

60. Takeuchi A, Saito T. CD4 CTL, a Cytotoxic Subset of CD4+ T Cells, Their Differentiation and Function. Front Immunol (2017) 8:194. doi: 10.3389/ fimmu.2017.00194

61. Glimcher LH, Townsend MJ, Sullivan BM, Lord GM. Recent Developments in the Transcriptional Regulation of Cytolytic Effector Cells. Nat Rev Immunol (2004) 4:900-11. doi: 10.1038/nri1490

62. Weiskopf D, Bangs DJ, Sidney J, Kolla RV, De Silva AD, De Silva AM, et al. Dengue Virus Infection Elicits Highly Polarized CX3CR1+ Cytotoxic CD4+ T Cells Associated With Protective Immunity. Proc Natl Acad Sci (2015) 112: E4256. doi: 10.1073/pnas.1505956112

63. Chen B, Morris SR, Panigrahi S, Michaelson GM, Wyrick JM, Komissarov AA, et al. Cytomegalovirus Coinfection Is Associated With Increased Vascular-Homing CD57(+) CD4 T Cells in HIV Infection. J Immunol (2020) 204:2722-33. doi: 10.4049/jimmunol.1900734

64. Chevalier Mathieu F, Jülg B, Pyo A, Flanders M, Ranasinghe S, Soghoian Damien Z, et al. HIV-1-Specific Interleukin-21+ CD4+ T Cell Responses Contribute to Durable Viral Control Through the Modulation of HIV-Specific CD8+ T Cell Function. J Virol (2011) 85:733-41. doi: 10.1128/JVI.02030-10

65. Harper J, Huot N, Micci L, Tharp G, King C, Rascle P, et al. IL-21 and Ifn $\alpha$ Therapy Rescues Terminally Differentiated NK Cells and Limits SIV Reservoir in ART-Treated Macaques. Nat Commun (2021) 12:2866. doi: 10.1038/ s41467-021-23189-7

66. Ahmad R, Sindhu Sardar TA, Toma E, Morisset R, Ahmad A. Elevated Levels of Circulating Interleukin-18 in Human Immunodeficiency Virus-Infected Individuals: Role of Peripheral Blood Mononuclear Cells and Implications for AIDS Pathogenesis. J Virol (2002) 76:12448-56. doi: 10.1128/JVI.76.24.1244812456.2002

67. Roy S, Balasubramanian S, Sumandeep S, Charboneau R, Wang J, Melnyk D, et al. Morphine Directs T Cells Toward T(H2) Differentiation. Surgery (2001) 130:304-9. doi: $10.1067 / \mathrm{msy} .2001 .116033$

68. Greeneltch KM, Kelly-Welch AE, Shi Y, Keegan AD. Chronic Morphine Treatment Promotes Specific Th2 Cytokine Production by Murine T Cells In Vitro via a Fas/Fas Ligand-Dependent Mechanism. J Immunol (2005) 175:4999. doi: 10.4049/jimmunol.175.8.4999

69. Hensley-Mcbain T, Zevin AS, Manuzak J, Smith E, Gile J, Miller C, et al. Effects of Fecal Microbial Transplantation on Microbiome and Immunity in Simian Immunodeficiency Virus-Infected Macaques. J Virol (2016) 90:49819. doi: 10.1128/JVI.00099-16

70. Benmebarek M-R, Karches CH, Cadilha BL, Lesch S, Endres S, Kobold S. Killing Mechanisms of Chimeric Antigen Receptor (CAR) T Cells. Int J Mol Sci (2019) 20:1283. doi: 10.3390/ijms20061283

71. Smith C, Elhassen D, Gras S, Wynn KK, Dasari V, Tellam J, et al. Endogenous Antigen Presentation Impacts on T-Box Transcription Factor Expression and Functional Maturation of CD8+ T Cells. Blood (2012) 120:3237-45. doi: 10.1182/blood-2012-03-420182

72. Banga R, Munoz O, Perreau M. HIV Persistence in Lymph Nodes. Curr Opin HIV AIDS (2021) 16:209-14. doi: 10.1097/COH.0000000000000686

Conflict of Interest: The authors declare that the research was conducted in the absence of any commercial or financial relationships that could be construed as a potential conflict of interest.

Publisher's Note: All claims expressed in this article are solely those of the authors and do not necessarily represent those of their affiliated organizations, or those of the publisher, the editors and the reviewers. Any product that may be evaluated in this article, or claim that may be made by its manufacturer, is not guaranteed or endorsed by the publisher.

Copyright $\odot 2021$ Olwenyi, Johnson, Pandey, Thurman, Acharya, Buch, Fox, Podany, Fletcher and Byrareddy. This is an open-access article distributed under the terms of the Creative Commons Attribution License (CC BY). The use, distribution or reproduction in other forums is permitted, provided the original author(s) and the copyright owner(s) are credited and that the original publication in this journal is cited, in accordance with accepted academic practice. No use, distribution or reproduction is permitted which does not comply with these terms. 